\title{
Décadrages Décadrages
}

cinéma, à travers champs Cinéma, à travers champs

29-30 | 2015

René Vautier

\section{Entretien avec René Vautier : les résonances d'Afrique 50}

François Bovier, Cédric Fluckiger et Elif Ugurlu

\section{(2) OpenEdition}

1 Journals

Édition électronique

URL : https://journals.openedition.org/decadrages/806

DOI : $10.4000 /$ decadrages.806

ISSN : 2297-5977

Éditeur

Association Décadrages

Édition imprimée

Date de publication : 1 avril 2015

Pagination : 163-167

ISBN : 9782970096306

ISSN : 2235-7823

\section{Référence électronique}

François Bovier, Cédric Fluckiger et Elif Ugurlu, «Entretien avec René Vautier : les résonances d'Afrique 50 », Décadrages [En ligne], 29-30 | 2015, mis en ligne le 01 mars 2017, consulté le 24 mars 2023. URL : http://journals.openedition.org/decadrages/806 ; DOI : https://doi.org/10.4000/decadrages.806

\section{cc) (i) (2) (2)}

Creative Commons - Attribution - Pas d'Utilisation Commerciale - Partage dans les Mêmes Conditions 4.0 International - CC BY-NC-SA 4.0

https://creativecommons.org/licenses/by-nc-sa/4.0/ 


\section{Entretien avec René Vautier : les résonances d'Afrique 50}

\begin{abstract}
Pour commencer, nous pourrions repartir d'Afrique 50 et de sa diffusion: quelle est l'actualité de ce film, quelles sont ses résonances aujourd'hui?

Quand on revoit un film que l'on a fait il y a longtemps, on se demande toujours si cela vaut la peine d'organiser de nouvelles projections. Peut-il avoir un écho, une influence positive aujourd'hui? Je pense que c'est le cas pour Afrique 5o. Le film a été interdit pendant très longtemps, ce qui prouve qu'il pouvait faire peur à certaines personnes. Et il est utile de le montrer encore à présent, les erreurs et les répressions des années 1950 ayant encore des répercussions politiques aujourd'hui.
\end{abstract}

En assistant aujourd'hui à une projection avec des jeunes qui n'ont pas vécu cette période, je vois que quelque chose les intéresse dans mon film, les fait réfléchir. C'est important, et pas seulement pour moi réalisateur, de se dire que l'on laisse quelque chose derrière soi: ce film, pendant des années, encore après un demi-siècle, n’a jamais cessé d'être utile; et malheureusement, il devient peut-être de plus en plus utile aujourd'hui à cause des résurgences du colonialisme des politiques mondiales. Ça réchauffe mes vieux os de me dire qu'en reflétant quelque chose, on n'a pas été totalement inutile. Je crois encore aujourd'hui qu'Afrique 50 est un film «vert»; c'est un film de jeune, j'avais 2I ans quand je l'ai fait. Il n'est pas devenu un film de vieux, et c'est réconfortant pour moi.
Une des raisons du caractère percutant, pertinent et actuel du film, tient aussi à sa forme. Le film emprunte la forme du tract, du manifeste; il interpelle directement le spectateur, malgré les soixante années qui se sont écoulées. Peux-tu nous dire comment tu as conçu sa forme?

C'est difficile pour un vieux réalisateur de retracer la genèse de son premier film; de retrouver, soixante ans après, pourquoi il a dit telle ou telle chose, en image, à telle ou telle période.

Après la guerre, les copains de résistance m’ont demandé ce que j’allais faire maintenant. Je leur ai dit que je voulais continuer à me battre contre les choses qu'il est nécessaire de changer. Mais pas avec une arme, pas en tuant. En faisant de la photo ou du cinéma, je ne savais pas encore. Et ce sont mes camarades du maquis qui m'ont dit: on va t'expédier à travers le monde et tu filmeras ce qui ne va pas et tu viendras nous le dire et nous le montrer. Et aussi, n'oublie pas qu'il y a des choses chez nous qui ne vont pas, il faudra que tu les montres aussi. Tu vas aller dans une école de cinéma. Tu vas nous représenter. Avec ce que tu apprendras dans cette école, tu nous représenteras, nous, groupe éclaireur de France de Quimper, cité à l'ordre de la nation pour faits de résistance. J'ai dit: d'accord, je veux continuer à me battre, mais avec une caméra.

Quand on m'a proposé de tourner un film sur un sujet que je ne connaissais pas, de faire un film 
sur «la vie réelle des Africains noirs», j’ai saisi cette opportunité. Je suis parti sans idée préconçue, et je me suis retrouvé dans une période de colonialisme virulent; et ce sont simplement mes réactions de jeune homme sortant de la période de la résistance, de lutte contre un occupant, l'occupant allemand en France; et je me suis rendu compte qu'en Afrique noire, les Français étaient aussi des occupants.

Ensuite, quand on a essayé de m'interdire de tourner ce que je voyais, cela m’a révolté, et je suis entré en dissidence. C'est-à-dire que j’ai choisi mon camp. Je me suis dit que j’allais filmer ce que les autorités coloniales voulaient m'empêcher de dénoncer. J'estimais ne pas trahir ce qu'on m'avait demandé de faire, c'està-dire un film sur la vie réelle des paysans africains, pour la Ligue de l'Enseignement. La vie de ces gens était intenable, et le colonialisme portait en lui-même les germes de sa pourriture. Et qu'on se serve d'un décret Pierre Laval pour m'interdire de filmer ce que je vois et ce qui me répugne, et bien cela a constitué une raison de plus pour que je le fasse. Alors, on m'a mis dans une résidence surveillée à Bamako, dont je me suis enfui.

C’est vrai qu'à ce moment-là, je me suis orienté vers un film contestataire dénonçant une situation politique existante. Je me suis donc retrouvé aux côtés de personnes qui se battaient déjà, dix ans auparavant, pour l'indépendance des peuples africains. J'ai fait ce film en tant que jeune résistant, en suivant les idées qui avaient été les miennes quand je me battais pour la résistance en France. J'ai continué à me battre pour la même cause en tournant Afrique 50.

J'étais aussi animé par cette idée: si on m’interdit de filmer des scènes, il faut à tout prix que je les montre. Et c'est ce que j’ai fait. Mais très rapidement, dans l'urgence, avec des gens qui organisaient leur lutte, avec les Africains. Avec ce besoin de dire: ce n'est pas parce que j'ai une caméra que je vais affirmer tout seul ce que je pense. Avec ma caméra, je vais filmer les gens à qui on interdit théoriquement de penser et donc de s'exprimer sur l'état dans lequel ils vivent. Ce sont peut-être eux, ces Africains, aux prémisses de leur indépendance, qui ont contribué à me former en tant qu'homme et cinéaste, plus que moi je n'ai participé à les former. Mais je pense aussi que j’ai apporté ma pierre à leur lutte.

\section{Quels ont été tes appuis à ton retour en France?}

Les autorités françaises savaient que j’avais tourné ce film. Elles savaient que j'étais de retour en France, et elles savaient aussi ce que j’allais dire dans ce film. J'ai donc donné à la Ligue de l'Enseignement les bobines. La ligue les a aussitôt remises à la police et nous avons commencé à les visionner en présence de deux policiers. C'était la première fois que je voyais ce que j’avais filmé, et je me suis dis qu'il fallait absolument le montrer.

Pendant la matinée, à la fin de la projection des bobines de trois minutes, la police saisissait chaque bobine. Après la pause de midi, je suis revenu avec des boîtes de bobines vides. Les projections ont redémarré l'après-midi. Mais cette fois, comme on ne rallumait pas entre les chargements de bobines, j’ai commencé à échanger les boîtes de bobines pleines par des bobines vides avant de les donner aux gendarmes. Je suis ressorti avec le tiers des bobines que j’avais tournées.

Jai donc pu commencer le montage à partir de ces bobines récupérées, avec des moyens rudimentaires, dans l'école maternelle où ma mère était institutrice - sous la protection des copains du quartier!

Comme tout le monde était au courant, quand les policiers sont venus pour saisir la pellicule, il y a eu une pression populaire pour les empêcher de péné- 
trer dans l'école. Trois cents personnes étaient là pour empêcher six policiers d'entrer dans les logements des instituteurs de l'école où je montais le film. J'ai pu donc terminer mon film sous la protection populaire.

Ensuite, le groupe de Keïta Fodéba est venu enregistrer la musique d'un bout à l'autre lors d'une projection en plein air dans le jardin de la maison des syndicats, avec quatre cents personnes qui assistaient à la projection. J'ai réalisé le commentaire dans les mêmes conditions. Et puis le film a existé sous la protection populaire, y compris lors de ses premières projections. C'est à ce moment-là que j’ai appris que j'étais recherché en vertu du décret Pierre Laval, ministre des Colonies, qui a été fusillé pour collaboration en 1945 .

C'est à partir de la diffusion d'Afrique 50 que je me suis rendu compte qu'en se battant publiquement contre la censure, on pouvait l'emporter, avoir sa peau. Peut-être que le tournage et la diffusion d'Afrique 50 m’ont mis sur les rails pour défendre aussi des films qui étaient faits par d'autres. C'était par exemple le cas pour un film tourné pendant la guerre d’Algérie, réalisé par Jacques Panigel, et qui n’avait pas de visa de censure. C'est un film composé d'entretiens sur la répression anti-algérienne d'une manifestation à Paris, où deux cents manifestants algériens ont été jetés dans la Seine par des policiers. J'ai entrepris une grève de la faim jusqu'à ce qu'on obtienne le visa pour Octobre à Paris et qu'on reconnaisse que censurer un film pour des raisons politiques est illégal. Au bout de 3r jours, un ministre a signé un papier... Mais déjà au bout du quinzième ou seizième jour, le film avait obtenu son visa.

Afrique 50 a aussi montré qu'on pouvait se battre collectivement contre la commission de censure et la notion même de censure. Il a marqué une date en France pour la liberté d'expression. Parler de l'inter- diction du film, oui; mais aussi de sa libération! Le film est encore projeté maintenant sans visa. J'ai refusé encore aujourd'hui de demander un visa. Par contre, il passe à la télévision. Mais jamais sur une chaîne française destinée aux Français. Il passe sur des chaînes qui montrent ailleurs à quel point la France était large d'esprit en acceptant de réaliser des films de cette sorte. Ce sont des programmes destinés à l'étranger...

Parmi différents épisodes humoristiques, j’ai été invité un jour à Beaubourg par le ministère des Affaires étrangères à la projection du film et à la remise d'une copie «de ce film courageux et nécessaire» dans une salle publique qui porte aujourd'hui le nom de Georges Pompidou, ancien président de la République. Alors que ce même Georges Pompidou défendait le droit à la censure et le droit à l'interdiction en disant: la télévision, qui est financée par le pouvoir, doit transmettre le point de vue du pouvoir. C’était sa définition des devoirs de la télévision publique en France.

\section{Comment conçois-tu aujourd'hui la diffusion du ci- néma militant?}

Théoriquement, on n'a pas le droit en France d'interdire un film - et ce, depuis nos luttes pour la liberté d'expression -, sauf si la commission de censure décrète qu'il faut l'interdire pour pornographie ou pour violence. Or maintenant, on n'interdit plus un film pour pornographie en France. Pour violence, c'est différent. La notion de lutte violente a servi face à Marée noire, colère rouge (1978), pour demander son interdiction. Mais le film a tout de même obtenu un visa d'exploitation. C'est un film qu'on avait fait avec les personnes qui se battaient contre la marée noire en France, et c'est un film qui a enclenché des luttes à travers le monde contre d'autres marées noires. La 
diffusion de ce film à travers le monde, accompagné d'autres films réalisés sur le même sujet, de jugement en jugement et de film en film, a fait naître une notion juridique, un droit dans le cas de marées noires: les pollueurs doivent être les payeurs. Mais sur ce même sujet, il y a encore des images qui sont interdites. Par exemple, des images ont été tournées sur les côtes libanaises pendant les bombardements de l'armée israélienne sur les dépôts de fuel. Il y donc eu une pollution provoquée par ces bombardements israéliens volontaires et ciblés. Aujourd'hui, il y a un combat à mener qui rejoint tous les combats populaires qu'on a pu lancer en réalisant ces films qui sopposaient parfois au pouvoir des gouvernements. On a à présent la possibilité de se servir d'images des dégâts causés par les naufrages passés des pétroliers et de les associer aux images actuelles de pollutions provoquées volontairement par un Etat, à travers les bombardements de l'armée israélienne. Est-ce que nous arriverons, comme nous l'avons obtenu grâce à nos films, à étendre la notion de "pollueurs payeurs» des compagnies pétrolières aux Etats, et d'obtenir ainsi leur condamnation? Là, se pose aussi la question suivante: peut-on exiger de projeter des films dans les pays où ils ont été tournés, alors que dans ces films on met en cause et on critique le gouvernement?

Il y a des gens qui me demandent aujourd'hui: «qu'est-ce que ça te fait de présenter Afrique 50 et de te remémorer tous les emprisonnements que tu as traversés en te battant à coup de films pour la liberté d'expression en France, pour la liberté d'expression par l'image et le son?» Les problèmes que j’ai pu avoir ne viennent pas entièrement des censeurs. Mais c'est vrai aussi que ça m'amuse d'en parler, ou de voir des films qui se font là-dessus. Par exemple le film L'Homme de la paix (I999) d’Ahcène Osmani, qui a obtenu le grand prix tolérance de l'UNESCO, n’a jamais été diffusé sur une chaîne de télévision française, mais pas plus en Algérie. Pourquoi?

Parce que dans ce film algérien, les réalisateurs donnent la parole à René Vautier. Et dans ce film, je raconte que pendant que j'étais en train de tourner un film sur les Algériens dans les maquis pendant la guerre d'Algérie, j’ai été arrêté et mis en prison pendant 25 mois. Car le gouvernement provisoire de la République algérienne et certains responsables algériens ne pouvaient pas dire que ce film, qu'ils considéraient comme le premier film algérien et qui était largement diffusé comme tel à l'étranger, avait été fait par un Français qui était communiste. En tant que communiste, je n'aurais pas dû mettre mon nom au générique; mais il n’y avait pas de générique! Donc, ils pouvaient diffuser le film mais il ne fallait pas que le réalisateur à ce moment-là dise que c'était lui qui l'avait tourné. Alors, il fallait me faire disparaître. Mes codétenus, tous algériens, sont devenus des amis et après leur libération des ministres pour certains d'entre eux. Ce sont eux qui ont demandé que ce soit moi qui forme les Algériens à faire des films libres. C'est-à-dire des films libres vis-à-vis du pouvoir algérien, comme j'en faisais vis-à-vis du pouvoir français pendant la guerre d'Algérie. Il y a une génération d'Algériens qui a été formée dans cette optique au Centre audiovisuel d'Alger. Et on a fait ensemble un film qui définissait ce que devait être l'avenir de l'Algérie sur le plan de la liberté d'expression. Ce film, Un peuple en marche (1963), sert encore aujourd'hui beaucoup aux Algériens pour les luttes pour leur liberté d'expression en Algérie.

Comme l'a dit l'un des premiers cinéastes africains formé à l'Institut des Hautes Etudes Cinématographiques (IDHEC) où j’avais étudié, Paulin Vieyra, qui, après avoir beaucoup travaillé avec Jean Rouch, 
était devenu responsable du cinéma dans la république sénégalaise indépendante: le film de Vautier, c'est le premier film africain. Et ça m’a fait très plaisir que des Algériens disent aussi: le premier film de Vautier tourné pendant la révolution en Algérie, c'est le premier film algérien. Et peut-être que les films que j'ai tournés aussi plus tard pour la Ligue des droits de l’homme dans le Pacifique seront aussi considérés par les néo-Calédoniens comme leurs premiers films indépendants.

Propos recueillis à Cancale, en 2009 